\section{Commentary: Dying with versus dying from hospital-acquired infections following cardiac surgery}

\author{
Harold L. Lazar, MD
}

In this edition of the Journal, Massart and colleagues ${ }^{1}$ report the mortality attributed to hospital-acquired infections (HAIs) following cardiac surgery in a retrospective study of 8853 patients. Although the incidence of HAI was only $4.2 \%$, when 370 patients with HAIs were compared with 370 patients without HAIs using propensity matching, hospital mortality was significantly greater in the patients with HAIs $(15.4 \%$ vs $5.7 \% ; P<.001)$. Infections with Pseudomonas aeruginosa, bloodstream infections, and pneumonia were independently associated with increased hospital mortality. The primary end point of the study was the "attributable mortality factor" due to HAI, which was defined as the proportion of death due to HAI and was calculated by comparing the difference in the number of deaths in patients who developed HAI with the number of deaths in the group of propensity-matched patients who did not develop HAI. This was calculated to be $17.1 \%$. The authors concluded that HAI following cardiac surgery is responsible for the increased mortality seen in these patients. However, is the attributable mortality fraction a reliable method to assess the cause of death in a retrospective study? Are these patients dying with versus dying from HAI? Unfortunately, there are several limitations with this study that do not allow us to answer this important question.

Because of the retrospective design of the study, and the multiple comorbidities present in the patients with HAIs, it is impossible to determine what the actual cause of death

\footnotetext{
From the Division of Cardiac Surgery, Boston University School of Medicine, Boston, Mass.

Disclosures: The author reported no conflicts of interest.

The Journal policy requires editors and reviewers to disclose conflicts of interest and to decline handling or reviewing manuscripts for which they may have a conflict of interest. The editors and reviewers of this article have no conflicts of interest.

Received for publication Aug 27, 2020; revisions received Aug 27, 2020; accepted for publication Aug 28, 2020; available ahead of print Sept 1, 2020.

Address for reprints: Harold L. Lazar, MD, 80 East Concord St, Boston, MA 02118 (E-mail: harold.1.lazar@gmail.com).

J Thorac Cardiovasc Surg 2022;163:2141-2

$0022-5223 / \$ 36.00$

Copyright (c) 2020 by The American Association for Thoracic Surgery

https://doi.org/10.1016/j.jtcvs.2020.08.092
}

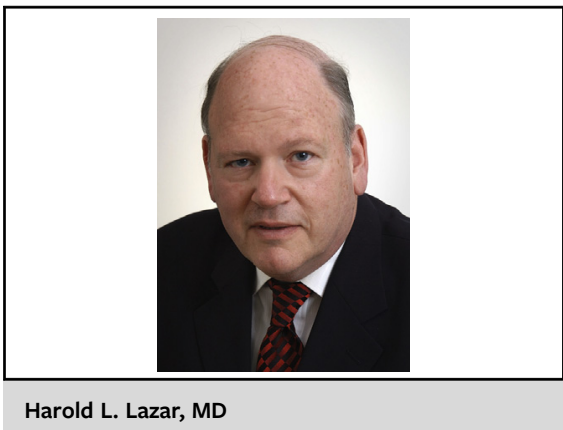

\author{
CENTRAL MESSAGE \\ Although mortality is increased \\ in cardiac surgical patients who \\ develop HAls, it remains unclear \\ as to whether these patients are \\ dying with versus dying from \\ these infections.
}

was in these patients. For example, if a patient is placed on an left ventricular assist device following surgery for postcardiotomy cardiogenic shock and fails to recover sufficient myocardial function such that a prolonged period of mechanical support is required during which time the patient develops pneumonia and sepsis and ultimately die, is this death related to postoperative cardiogenic shock or due to HAI? Clearly, the primary cause of death is persistent low cardiac output unresponsive to left ventricular assist device therapy. The same conclusion can be made for patients who develop postoperative renal failure and require renalreplacement therapy and die to sepsis due to an indwelling dialysis catheter, patients with a massive stroke who require prolonged ventilator support and die from pneumonia, and patients who develop postoperative cardiac tamponade, experience a period of low cardiac output, and develop multiorgan failure and ultimately die from gram-negative sepsis due to a perforated viscus. Unfortunately, the authors do not provide us with the incidence of these postoperative complications, which may have been responsible for the development of an infectious complication.

There are several other issues with this study that limit the conclusions that can be made regarding the contribution of HAI to mortality following cardiac surgery. The antibiotic coverage received by these patients is not clearly defined. We are only told that cefuroxime was given at the start and every 2 hours during the procedure. It appears that the Society of Thoracic Surgeons guidelines for antibiotic therapy in the perioperative period were not in 
place. ${ }^{2,3}$ In addition to the duration for perioperative antibiotics, these guidelines outline the indications for the addition of vancomycin in those patients who may be at greater risk for methicillin-resistant Staphylococcus aureus and stipulate the use of antibiotics for gram-negative coverage when vancomycin is the sole prophylactic antibiotic due to allergies to cephalosporins. This could have limited the incidence of $P$ aeruginosa infections seen in this study. Ventilator-associated pneumonias are a major cause of HAI; however, the authors fail to tell us what protocols were in place to prevent this complication. This would include broadening antibiotic coverage to treat $\mathrm{Paer}$ uginosa in patients requiring prolonged ventilator support; considering early tracheostomy in these patients to improve mobilization and clear secretions; and optimizing nutritional support.

The most glaring limitation of this study is the lack of information on glycemic control. Optimizing glycemic control in patients post-cardiac surgery has been shown to decrease the incidence of infectious complications, especially sternal wound infections. ${ }^{4}$ Van den Berghe and colleagues ${ }^{5}$ have shown that in those patients who require $>3$ days of care in the intensive care unit (ICU) due to ventilator, inotropic, or mechanical support, renalreplacement therapy, or the need for antiarrhythmic agents, more intensive glycemic control resulted in a significant decrease in mortality in these high-risk patients. The Society of Thoracic Surgeons guidelines for glycemic control in cardiac surgery patients recommend that glucose levels in the ICU be $<180 \mathrm{mg} / \mathrm{dL}$ and $<150 \mathrm{mg} / \mathrm{dL}$ in those high-risk ICU patients requiring ventilator, inotropic, and mechanical support. ${ }^{6}$ In addition, no mention is made by the authors as to whether the American Association for Thoracic Surgery guidelines for the prevention and treatment of sternal wound infections were also in place, which have been shown to significantly decrease the incidence of all types of sternal wound infections. ${ }^{\text {? }}$

The authors should be encouraged to build on their database and perform a prospective, observational study with appropriate guidelines in place for antibiotic coverage, glycemic control, and management of patients requiring prolonged ventilation to determine the actual cause of death in patients who develop an HAI. Only then can we determine whether patients are dying with versus dying from HAI following cardiac surgery.

\section{References}

1. Massart N, Mansour A, Ross JT, Piau C, Verhoye J-P, Tattevin P, et al. Mortality due to hospital-acquired infection after cardiac surgery. J Thorac Cardiovasc Surg. 2022; 163:2131-40.e3.

2. Edwards FH, Engelman RM, Houck P, Shahian DM, Bridges CR. The Society of Thoracic Surgeons practice guidelines series: antibiotic prophylaxis in cardiac surgery. Part I: duration. Ann Thorac Surg. 2006;81:397-404.

3. Engelman R, Shahian D, Shemin R, Guy TS, Bratzler D, Edwars F, et al. The Society of Thoracic Surgeons practice guideline series: antibiotic prophylaxis in cardiac surgery. Part II: antibiotic choice. Ann Thorac Surg. 2007;83:1569-77.

4. Lazar HL, Chipkin SR, Fitzgerald CA, Apstein CA. Tight glycemic control in diabetic coronary artery bypass graft patients improves perioperative outcomes and decreases recurrent ischemic events. Circulation. 2004;109:1497-502.

5. Van den Berghe G, Waters P, Weekers F. Intensive insulin therapy in critically ill patients. N Engl J Med. 2001;345:1359-67.

6. Lazar HL, McDonnell M, Chipken S, Furnary AP, Engelman RM, Sadhu AR, et al. The Society of Thoracic Surgeons practice guideline series: blood glucose management during adult cardiac surgery. Ann Thorac Surg. 2009;87:663-9.

7. Lazar HL, VanderSalm T, Engelman R, Orgill D, Gordon S. Prevention and management of sternal wound infections. J Thorac Cardiovasc Surg. 2016;152: 962-72. 